\title{
Screening radiographs for new adult patients
}

\author{
Screening panoramic radiography of new adult patients: diagnostic yield when combined with bitewing radiography \\ and identification of selection criteria V. E. Rushton, K. Horner, H. V. Worthington Br Dent J 2002; 192: 275-279
}

\section{Objectives}

To measure the radiological diagnostic yield on screening panoramic radiographs taken of new adult patients and to identify selection criteria for panoramic radiography of new adult patients.

\section{Design}

Survey of 1,817 consecutive panoramic radiographs taken as 'routine' on new patients with statistical analysis of clinical and radiological findings.

\section{Setting}

All radiographs were obtained from 41 general dental practitioners (GDPs). The GDPs provided the clinical information about the patient obtained by history and examination. Collection of material occurred in 1998/1999.

Materials and methods

Two dental radiologists recorded the radiological findings on each of 1,817 panoramic radiographs by consensus. Those findings that would have been identified from bilateral posterior bitewing radiographs of each patient were then excluded to give modified figures for radiological findings. Indices of diagnostic yield were devised and calculated for each radiograph from the data on radiological findings. Total diagnostic yield (DY) and modified diagnostic yield (MDY), after exclusion of findings identifiable on bitewing radiographs, were both calculated. Clinical indicators of a high MDY were identified using stepwise multiple regression analysis.

Results

MDY was 0 for 17\% of the radiographs (all patients) and 23\% of the radiographs when the asymptomatic group were considered. The clinical variables for which the significance was high $(p<0.001)$ were: increasing number of teeth with clinical suspicion of periapical pathology, partially erupted teeth, increasing number of clinically evident carious lesions, partially dentate status and crowns.

\section{Conclusion}

Taking posterior bitewing radiographs of new adult patients would reduce the diagnostic yield identified solely by panoramic radiography. Using clinical factors derived from the history and examination as radiographic selection criteria modestly improves the odds of achieving a high diagnostic yield from panoramic radiography.

\section{IN BRIEF}

- While panoramic radiography is frequently used as a routine 'screening' examination of a new adult patient, taking posterior bitewing radiographs, in agreement with current evidence-based clinical guidelines, reduces the numbers of radiological findings and the diagnostic yield identified solely by the panoramic radiograph.

- In asymptomatic patients, radiological diagnostic yield from panoramic radiographs is lower.

- Selecting patients for panoramic radiography using clinical factors derived from the history and examination would improve the odds of achieving a high diagnostic yield.

- Alternatives to panoramic radiography (posterior bitewings and selected periapicals) can be satisfactorily identify abnormalities with greater diagnostic accuracy.

\section{COMMENT}

This paper is published at an appropriate time, as new ionising radiation regulations have recently come into force in the UK which require that radiographic examinations should have a net benefit for the patient, and should normally provide new information to aid the patient's management or prognosis. This raises the question of the ethics of screening radiographs, as used in this study.

This study looked at a large number $(1,817)$ of 'screening' radiographs taken in general dental practice. The aims were to measure the radiological diagnostic yield of screening panoramic radiographs taken of new adult patients and to identify selection criteria for panoramic radiography of new adult patients. It also specifically addresses the issue of whether the panoramic yield obtained is duplicating that which would be evident on intra-oral radiography at a lower or comparable $\mathrm{x}$-ray exposure to the patient. This is relevant as selection criteria in the UK indicate that posterior bitewing radiographs are recommended for the assessment of new adult dentate patients.

The radiological findings were recorded by two experts working together using a standard report form, and included details related to the supporting structures as well as the teeth; actual numbers of teeth with caries or periapical bone change were recorded. Clinical information was available from the dentists for all patients.

In order to provide numerical data that could be subjected to a statistical analysis, the findings were then summed in two ways: DY the diagnostic yield relates to all positive findings on the panoramic radiograph. MDY the modified diagnostic yield relates only to positive findings that would not have been evident on a bitewing radiograph. Within each of these groups there were two sub-groups: DY1/MDY1 each positive finding was given a score of one, eg five carious lesions $=5$. DY2/MDY2 each category of positive findings was given a score of one, eg five carious lesions $=1$

The results were correlated with the clinical findings and it was found that patients with a 'high' diagnostic yield had: not visited the dentist for a longer time, significantly more restorations, significantly poorer oral hygiene, significantly more teeth with suspected periapical pathology and were less likely to be fully dentate. This finding seems to support the radiographic investigation but in the discussion the author recognises that positive findings may be identified and yet require no intervention (eg an unerupted third molar).

The analysis of the results has led the author to conclude that application of selection criteria for intra oral radiography could effectively remove any perceived need for panoramic radiography for the majority of patients, and she draws the conclusion that the use of panoramic radiographs in general dental practice can be questioned when valid alternatives are readily available at lower costs and which improve diagnostic accuracy. These are sensible, evidence-based conclusions, and indicate that dentists should be using positive clinical findings as the basis for radiography selection criteria, and where possible using small numbers of intra orals when these can be considered to demonstrate the expected pathological changes.

Laetitia Brocklebank, Senior Lecturer/Hon. Consultant in Oral Radiology, Glasgow Dental Hospital and School 